ACCEPTED MANUSCRIPT

\title{
Development of polymeric anepectic meshes: auxetic metamaterials with negative thermal expansion
}

To cite this article before publication: Joana Santos Raminhos et al 2019 Smart Mater. Struct. in press https://doi.org/10.1088/1361-665X/ab034b

\section{Manuscript version: Accepted Manuscript}

Accepted Manuscript is "the version of the article accepted for publication including all changes made as a result of the peer review process, and which may also include the addition to the article by IOP Publishing of a header, an article ID, a cover sheet and/or an 'Accepted

Manuscript' watermark, but excluding any other editing, typesetting or other changes made by IOP Publishing and/or its licensors"

This Accepted Manuscript is @ 2019 IOP Publishing Ltd.

During the embargo period (the 12 month period from the publication of the Version of Record of this article), the Accepted Manuscript is fully protected by copyright and cannot be reused or reposted elsewhere.

As the Version of Record of this article is going to be / has been published on a subscription basis, this Accepted Manuscript is available for reuse under a CC BY-NC-ND 3.0 licence after the 12 month embargo period.

After the embargo period, everyone is permitted to use copy and redistribute this article for non-commercial purposes only, provided that they adhere to all the terms of the licence https://creativecommons.org/licences/by-nc-nd/3.0

Although reasonable endeavours have been taken to obtain all necessary permissions from third parties to include their copyrighted content within this article, their full citation and copyright line may not be present in this Accepted Manuscript version. Before using any content from this article, please refer to the Version of Record on IOPscience once published for full citation and copyright details, as permissions will likely be required. All third party content is fully copyright protected, unless specifically stated otherwise in the figure caption in the Version of Record.

View the article online for updates and enhancements. 


\section{DEVELOPMENT OF POLYMERIC}

\section{ANEPECTIC MESHES: AUXETIG METAMATERIALS WITH} NEGATIVE THERMAL EXPANSION

\section{Authors}

J. S. Raminhos ${ }^{1}$, J. P. Borges ${ }^{1}$, A. Velhinho ${ }^{1}$

\section{Affiliations}

${ }^{1}$ CENIMAT/I3N, Departamento de Ciência dos Materiais, Faculdade de Ciências e Tecnologia, FCT, Universidade Nova de Lisboa, 2829-516, Campus de Caparica, Portugal

\section{Contact information}

J. S. Raminhos, j.raminhos@ campus.fct.unl.pt

J. P. Borges, jpb@fct.unl.pt (corresponding author)

A. Velhinho,ajv@fct.unl.pt (corresponding author) 


\section{Abstract}

This paper reports the application of additive manufacturing technology to fabricate bidimensional lightweight composite meshes capable of demonstrating auxetic properties (negative Poisson's ratio (NPR)) in combination with negative thermal expansion (NTE) behaviour, using as constituent materials polymers that do not exhibit NTE behaviour. To describe the combination of NPR and NTE characteristics, the designation of 'anepectic' is being proposed. Each mesh, obtained from varying either the material combination or the design parameters, was tested on a heated silicone bath to study the effects of the different combinations on the coefficient of thermal expansion (CTE). It was found that all meshes studied demonstrated a successful combination of NPR and NTE behaviours, and it was revealed that there is a possibility to tailor the meshes to activate the NTE behaviour within a chosen range of temperatures. For an extreme case, a Poisson's ratio of -0.056 , along with a CTE of $-1568 \times 10^{-6} \mathrm{~K}^{-1}$ has been achieved.

\section{Keywords}

3-D printing, Anepectic, Auxetic, Negative thermal expansion, Negative Poisson's ratio, Tuneable thermal expansion 


\section{Introduction}

Within the context of the present work, the authors propose the designation "Anepectic". This derives from the Greek root 'E $\pi \varepsilon ́ \kappa \tau \alpha \sigma \eta '$ ('Epéktasi'), meaning expansion, duly affected by the negative prefix to designate materials capable of demonstrating negative Poisson's ratio (NPR) and, simultaneously, negative thermal expansion (NTE) behaviour. Materials with these combined behaviours are starting to be explored by the scientific community [1]-[3].

When subject to thermal change, most materials contract when cooled and expand when heated because the rising temperature induces the elongation of interatomic bonds that manifests itself at the macro-scale as volume expansion. Nonetheless, some solids contract with raising temperatures, exhibiting NTE [4]-[6]. The behaviour these solids present is especially useful for applications where the disparity in thermal stress should be carefully managed, such as precision instruments, high precision optical devices [7], [8], satellite antennas, thermally stable space telescope mirrors [9], [10], microchip devices [7], [10], adhesive fillers, dental filling composites [11], fibre optics [8], bridges, space vehicles [12], which undergo environmental conditions with variable temperatures. For such applications, materials with NTE characteristics offer a possibility for tailoring thermal expansion, thus moderating thermomechanical stresses, mitigating thermal damage and consequently enhancing equipment reliability and prolonging its lifetime over a wider range of operating temperatures. Additionally, such materials would allow for the production of biomedical devices such as stents, surgical hernia meshes (SHM), compression garments and other applications, presenting many advantages over current designs.

Materials with NPR get shorter (longer) in transverse direction/when compressed (tensioned) along the longitudinal direction. Negative values of Poisson's ratio are not just hypothetical, but they can be achieved in man-made materials, designated auxetic materials. These structures exist in many different scales: from the micro-structural and molecular to the macroscopic scale. Materials with this unusual behaviour are gaining interestin multiple areas [13].

Even though some prior mentions to the possibility of orthotropic materials showing negative values of Poisson ratio are available [14], [15], [16], [17], the pioneer researcher in the field of negative Poisson's ratio isotropic materials was Lakes, who in 1987 published one of the earliest known publications about this topic [18], but the term auxetic first appeared in a 1991 paper by Evans et al. [19]. It comes from the Greek $\alpha \hat{\xi} \xi \eta \tau \iota \kappa o ́ \varsigma$ (auxetikos), translated to "which tends to increase" and having its root in the word $\alpha$ w $\xi \eta \sigma \varsigma$, or auxesis, signifying "an increase".

Auxetics can be considered metamaterials, which are materials that are artificially engineered to gain emerging properties and functionalities otherwise unattainable in natural materials. Their performance and behaviour are a direct consequence of the design of their inherent and specific spatial arrangement, rather than material composition, therefore they are organized in patterns structured with precise shape, geometry, size and orientation. The deformation mechanisms of auxetics depend on their hinge-like structure, which bends outwards when stretched.

To create lightweight micro-architectured systems with interesting mechanical properties, the auxetic behaviour has been stúdied in cellular structures, and 2-D and 3-D architectures have been showed to possess auxetic behaviour in micro- and macro-scale [20]-[25]. Many approaches have been explored to design auxetic metamaterials (two-fold Miura-Ori [26], elastic buckling [27]) and auxetic behaviour has been reported in crumpled aluminium thin foils [28], two-phase honeycomb structures [29], three-dimensional chiral lattices [30], entangled single wire materials [31], and slitted sheets with perforations patterned according to a hexagonal periodicity [32]. There are also examples of composite materials with no voids within their internal structure which exhibit auxetic behaviour [33]. Furthermore, temperature has been used as a control parameter of the auxetic behaviour [34], [35]. 
Previously mentioned cellular auxetic structures are fabricated using advanced 3-D printing/additive manufacturing techniques. The complexity of the aforementioned structures inexorably leads to the use of polymeric 3-D printers that now feature the possibility of printing with multiple materials, giving way to fairly accessible, more complex structures with remarkable properties. An auxetic dual metamaterial was manufactured with a new polymeric 3-D printer, capable of dual-material fabrication [36]. It was concluded that the material selection and fraction of stiff regions can serve as important parameters in tuning auxetic response, by tailoring the relative stiffness of the component beams within the structure, it is possible to design an auxetic truss structure with a specific Poisson's ratio, shear modulus, and tensile modulus.

Inspired by the molecular NTE behaviour in bulk solids, there have been attempts to desígn NTE structures with flexible micro- or macro-architectures of periodic lattice units, by integrating components with distinct coefficients of thermal expansion (CTE) within its structures. The structural interaction between these constituents' prompt part of the structure to rotate or bend, to accommodate their varied thermal expansion within the internal free space available inducing overall volume contraction, thus leading to designed deformation. NTE structures can be tuned for a broad range of temperature by controlling the CTE of the constituent materials and the geometric layout of the structure [37]. This approach has been furthered by Grima et al. [38], which have imagined a pathway to obtain a variety of negative properties from structures built from bi-material ligaments. Simulation results have shown that some of these structures can exhibit anepectic properties [39].

Existing metamaterials with NTE results, using micro-architectured structures, have been mostly limited to structures with two-dimensional layouts [1], [40]-[46]. Only a few 3-D microstructures have been developed for such metamaterials [12], [37], [41], [45], [47], [48]. The inherent difficulty is mainly due to the limitations in fabricating three-dimensional multi-material structures with highly sophisticated geometric patterns. In addition, existing NTE structures were built with only limited material choices, therefore NTE could not be tuned within a pre-selected range of temperatures [49]-[51].

Metallic structures can be fabricated from metal powders using electron beam melting (EBM), selective laser sintering, laser ablation methods, and lithography [37], [52]. However, the literature also suggests that materials based on polymer bi-layers can deliver higher thermal expansion than those based on metallic bi-layers [43], a consideration which provided motivation for the current work.

There have been discussions of 2-D lattice-based metamaterials with simultaneous NPR and NTE behaviour [53]. However, prior studies have failed to deliver such structures physically, even though the researchers discuss the laser-based "printability" of 3-D metallic metamaterials. The approach for most studies has been to simulate metamaterials with the desired behaviours through finite element modelling, concluding the study with a virtual solution which, if fabricated, may achieve both NPR and NTE while maintaining a high stiffness and a low relative density (making it lightweight).

In most cases, meshes made from two different types of materials are proposed. The requirement for these materials is that they must be of similar stiffness but widely differing thermal expansion [54]. In this manner, combining an inherently auxetic structure with a dualmaterial NTE mesh design it has been suggested that an anepectic metamaterial may be achievable.

However, little research has been conducted to achieve the physical embodyment of such solutions, and this circumstance provided further motivation for the current work, through the opportunity to fabricate the first non-virtual anepectic meshes. Polymer constituent materials, with positive intrinsic CTE, were used for the fabrication of the meshes. In accordance with 
criteria based on those proposed for metallic materials by L. Ai et al. [53], in the case of similar (yet different) stiffness but widely differing thermal expansion, a stiffer constituent material that simultaneously undergoes the smallest dilation upon heating, should enforce a deformation on the other constituent material, characterized by being more flexible but having a higher CTE. By changing the relation between the Young's moduli and the CTE of the constituent materials, as well as the geometrical parameters of the mesh, it is hypothesised that it is possible to tune the behaviour of the resulting structure in order to trigger the anepectic behaviour in a chosen range of temperatures.

In this study, three main bi-dimensional meshes were designed, printed and tested. Their fabrication did not involve pins, adhesives, welding or pressure-fit joins. No alterations were done to the structures after their fabrication and they were tested as recovered from the printer. A commercially available 3-D printer has been used to print polymeric filaments, also available commercially. The meshes were evaluated to determine their CTE and Poisson's ratio. Combinations of four constituent materials were considered, copolyester (CPE+), Nylon, polypropylene (PP) and polyvinyl alcohol (PVA). Comparative studies about each different mesh were performed, regarding to their CTE and Poisson's ratio, and the relation between each variation is explored. Based on these values, all meshes studied demonstrated successful anepectic behaviour.

To the authors' knowledge, the current paper presents for the first time the production of a polymer-based composite mesh exhibiting anepectic behaviour, created from constituents with positive thermal expansion. These meshes may be applied to the biomaterial field and shall be tuned to work at the working temperature range of the human body.

\section{Mesh Design}

The meshes studied are based in a star-shaped re-entrant structure; such re-entrant structures are characterised by being inherently auxetic [24], [55], [56].

Figure 1 shows the main design behind all the meshes, the blue beams representing the material with lower CTE and higher Young's modulus, while the red beams correspond to the material with higher CTE and lower Young's modulus, as for the above stated criteria base on [53]. Each mesh structure is characterized by being star-shaped and of re-entrant design, with the same repeating unit cell throughout the entire mesh. The unit cells are characterized by using four geometrical parameters that retain the same meaning in every mesh: three length parameters $\left(\mathrm{H}_{1}\right.$ and $\mathrm{H}_{2}$, length parameters as defined per the scheme in figure 1 , and $\mathrm{t}$, which simultaneously represents the beams' thickness and width) and one angle parameter $(\theta)$. The re-entrant geometry of the meshes, responsible for the auxetic behaviour, was left undisturbed.

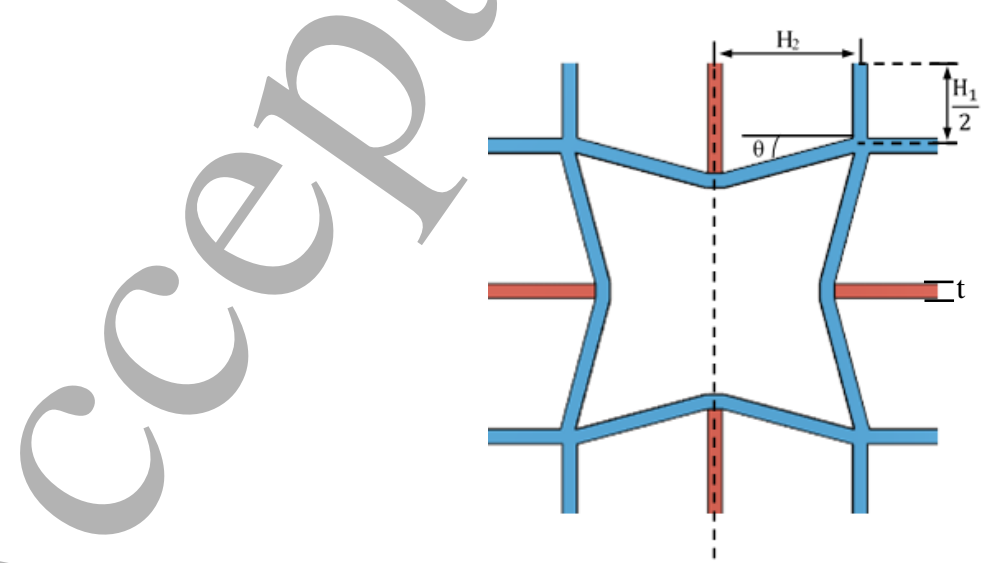

Figure 1 Unit cell that is repeated for the entirety of the mesh. Variation of the four geometrical parameters offers the possibility to modify the geometry of the meshes 
Figure 2 shows the five periodic mesh structures designed and studied. The cross-section of the beams is $1 \mathrm{~mm} \times 1 \mathrm{~mm}$ thick for every main mesh design (mesh \#1, mesh \#2 and mesh \#3) and of $0.5 \mathrm{~mm} \times 0.5 \mathrm{~mm}$ for the secondary meshes (mesh \#2 50\% and mesh \#3 50\%). Each mesh is symmetrical, and their geometrical parameters vary slightly form one mesh to the other, according to Table 1 .

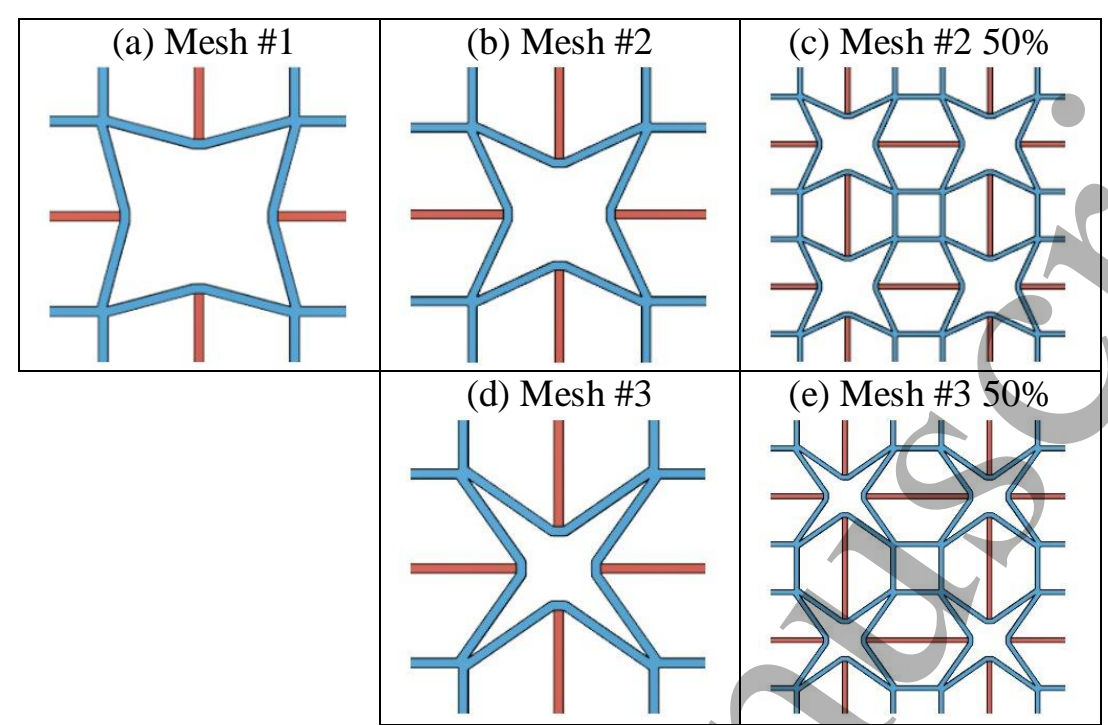

Figure 2 Five metamaterial meshes designed: (a) mesh \#1, (b) mesh \#2, (c) mesh obtain by scaling mesh \#2 down by $50 \%$, (d) mesh \#3, (e) mesh obtain by scaling mesh \#3 down by $50 \%$. A common scale was used for all the above representations.

\section{Method}

Table 1 Design parameters adopted for the meshes tested in this work.

\begin{tabular}{r|cccc}
\hline Parameters & $\begin{array}{c}\mathrm{H}_{1} \\
(\mathrm{~mm})\end{array}$ & $\begin{array}{c}\mathrm{H}_{2} \\
(\mathrm{~mm})\end{array}$ & $\begin{array}{c}\theta \\
\left({ }^{\circ}\right)\end{array}$ & $\begin{array}{c}\mathrm{t} \\
(\mathrm{mm})\end{array}$ \\
\hline Mesh \#1 & 10 & 10 & 15 & 1.0 \\
Mesh \#2 & 10 & 10 & 25 & 1.0 \\
Mesh \#2 50 \% & 5.0 & 5.0 & 25 & 0.5 \\
Mesh \#3 & 10 & 10 & 35 & 1.0 \\
Mesh \#3 50\% & 5.0 & 5.0 & 35 & 0.5
\end{tabular}

\subsection{Printing equipment and filament characterization}

The materials used are commercially available Ultimaker ${ }^{\mathrm{TM}}$ filaments of CPE+ (copolyester; black), Nylon (Polyamide (grade based on PA6/PA66); black), PP (Polypropylene; undyed) and PVA (Polyvinyl alcohol compound; undyed), adequate for processing with a commercial Ultimaker $3^{\text {TM }}$ fused filament 3-D printing-system. The two combinations selected for the current study are Nylon-PVA, and PP-CPE+.

Dogbone specimens for the mechanical characterization (elastic modulus) of 3D printed samples were designed. All the specimens were printed directly on the heating plate without any supports. The filling density of the specimens was set to $100 \%$, which means that all the specimens are solid structures. Tensile strength tests of different samples were carried out, the specimens being loaded to fracture $(0.5 \mathrm{~mm} / \mathrm{min})$ by using a universal testing machine (AG50kNG, Shimadzu, Japan). The glass and melt temperatures were obtained by differential scanning calorimetry (DSC 204 F1 Phoenix, Netzsch, Germany) extending from the temperature range of 223 to $523 \mathrm{~K}$, with a protective atmosphere using nitrogen gas and cooled with liquid 
nitrogen. The CTE of the materials was determined by thermo-mechanical analysis (TMA PT 1600, Linseis, Germany), the initial length of the specimens was of $10 \mathrm{~mm}$ and the analysis temperature range was from $-263 \mathrm{~K}$ to $338 \mathrm{~K}$, with no protective atmosphere. The individual constituents of the meshes all have positive coefficients of thermal expansion.

The mechanical and thermal characteristics determined for each material used in the course of this study are shown in Table 2.

Table 2 Material properties for CPE+, Nylon, PP and PVA.

\begin{tabular}{rccc}
\hline Material & $\begin{array}{c}\text { Young's modulus } \\
(\mathrm{GPa})\end{array}$ & $\begin{array}{c}\mathrm{CTE} \\
\left(\times 10^{-6} \mathrm{~K}^{-1}\right)\end{array}$ & $\begin{array}{c}\mathrm{T}_{\mathrm{g}} \\
(\mathrm{K})\end{array}$ \\
\hline CPE+ & 1.031 & 71 & 378 \\
Nylon & 0.889 & 166 & 308 \\
PP & 0.152 & 248 & 248 \\
PVA & 2.328 & 21 & 308
\end{tabular}

\subsection{Evaluation of thermo-mechanical characteristics of meshes}

The fabrication of the meshes was performed with the same Ultimaker $3^{\mathrm{TM}} 3$-D printingsystem mentioned above, which can process two different materials simultaneously. Subsequent mesh testing could be performed without the need for further finishing techniques, such as cleaning or polishing. For testing and analysis purposes small white marker dots were made on strategic locations on the mesh with white paint followed/by a dot of black permanent marker in the centre of each white dot, as a way of improving optical contrast in the photographs, in view of dimensional change measurements.

The auxetic capabilities of the meshes were evaluated at room temperature, through a tensile effort applied to induce a constant elongation rate of $1 \mathrm{~mm} / \mathrm{s}$ up to $10 \mathrm{~mm}$ overall elongation. To insure homogenous gripping at both ends of the mesh, a $10 \mathrm{~mm}$ wide support was printed to allow mounting the sample to a metallic support for mechanical testing. The metallic support at both ends was friction fastened to the tension grips of a Hounsfield $\mathrm{H} 5 \mathrm{~K}-\mathrm{W}$; during these tests the applied force was not recorded. Each sample was tested in a horizontal setup, to facilitate the measuring process. Each mesh was monitored using a digital SLR camera (12.2 M Pixel Canon $1100 \mathrm{D}$ with an EF-S 18-55mm lens) and photographs were taken at a rate of 1 photograph $/ \mathrm{mm}$. To limit parallax errors, the camera was mounted at a $90^{\circ}$ angle, directly above the thermal testing assembly. The photographs were analysed with ImageJ and the distances (measured in pixels), between the white dots on the meshes, led to the determination of the Poisson ratio, in accordance with Equation 1 below, where $\Delta y$ means elongation in the transverse direction, $y_{0}$ is the original length in the same direction and $\Delta x$ and $x_{0}$ are the corresponding values measured along the longitudinal direction:

$$
v_{x y}=-\frac{\varepsilon_{y}}{\varepsilon_{x}}=-\frac{\ln \left(1+\frac{\Delta y}{y_{0}}\right)}{\ln \left(1+\frac{\Delta x}{x_{0}}\right)}
$$

(Equation 1)

CTE testing, represented in figure 3, was conducted in a vat filled with silicone oil (Baysilone M350), in which a plate of $3 \mathrm{~mm}$ thick glass lined the bottom. The meshes were first submerged in room temperature silicone oil, after which a gridded ( $3 \mathrm{~mm}$ thick) glass plate was placed on top of the meshes. The gridded glass plate possessed $3 \mathrm{~mm}$ metallic spacers in the corners, to limit the out-of-plane motion of the mesh being tested. 


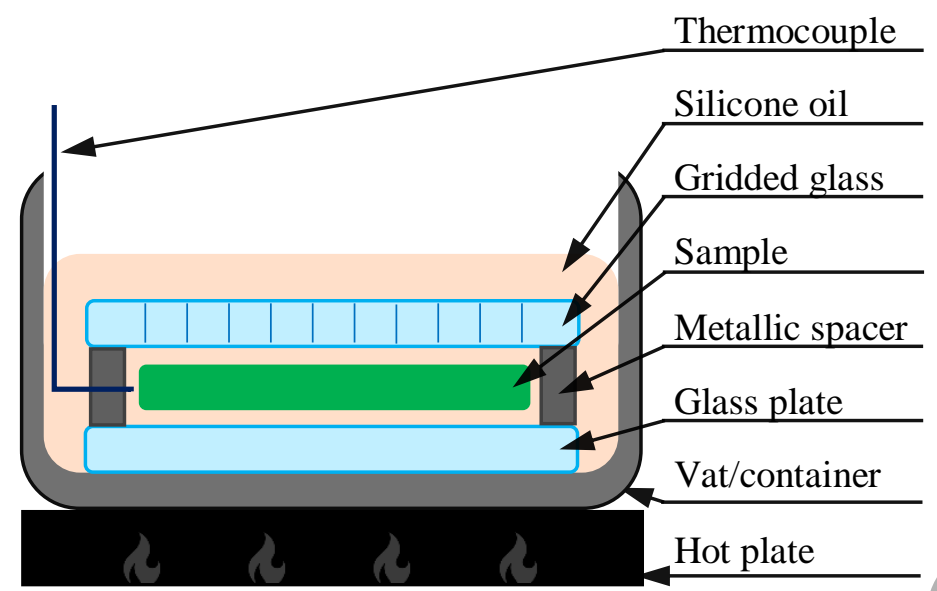

Fig. 3 Schematic representation and description of the testing structure performed.

The mesh was allowed to stabilise for 10 minutes, after which the silicone oil was heated, with the help of a hot plate, at a rate of approximately $3 \mathrm{~K} / \mathrm{min}$, until a set temperature $(358 \mathrm{~K}$ or, in some selected cases, $393 \mathrm{~K}$ ) was reached. The temperature of the silicone immersion was monitored through an external thermocouple and data logger (Pico TC-08 with a Type K thermocouple). A camera setup similar to that described for the auxetic behaviour measurements was used, directly above the mesh, at a $90^{\circ}$ angle, in order to capture the results, and photographs were taken at a rate of 1 photograph/K. As above, the photographs were analysed with ImageJ for determination of the CTE. This was established by comparison between images obtained at the set temperature and those registered at room temperature, at the start of the experiment.

Some meshes were subject to consecutive heating and cooling cycles. In those cases, the CTE values were determined at the high temperature at the end of each cycle; the resulting overall permanent deformation, however, could only be measured at the end of the last cycle.

In possession of the data from Table 2, a set of sample meshes was selected for the study, as per Table 3. The material selection was performed within the constraints set by the available set of printable filaments. Concerning the dual-material cases, the Nylon-PVA pair has been chosen to comply with the previously mentioned criteria (stiffer and less thermally expansive blue struts vs. more flexible and more thermally expansive red struts), with the PP-CPE+ pair standing for a similar situation, yet with a more dissimilar stiffness and a closer thermal expansion between the two materials.

Table 3 Summary of meshes considered for the present work. $\mathbf{x}$, not tested. $\checkmark$, tested.

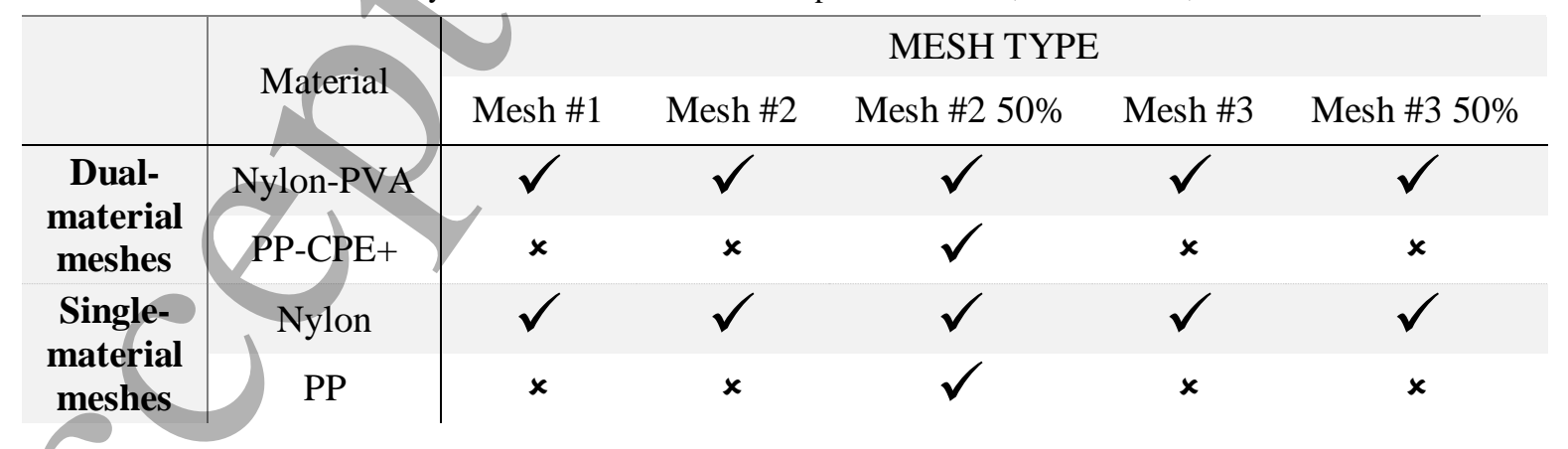

Figure 4 shows the meshes subject to experimentation, with the exception of Mesh \#2 50\% in $\mathrm{PP}$, due to a lack of contrast of the corresponding photographic record. 


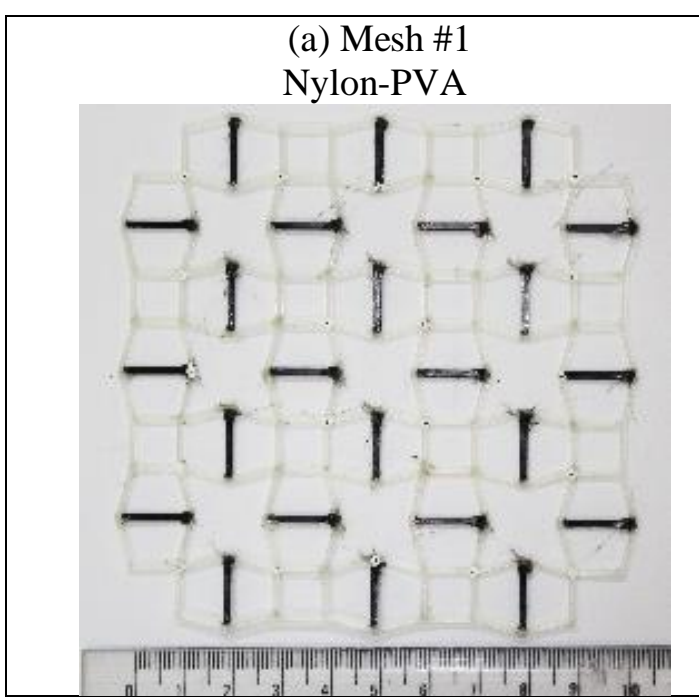

(c) Mesh \#2 Nylon

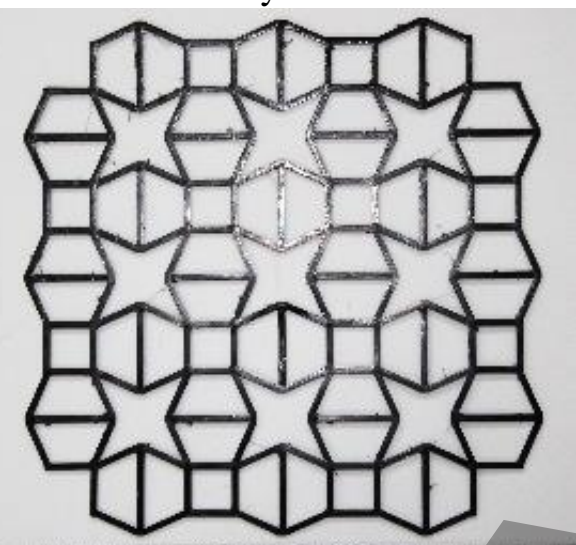

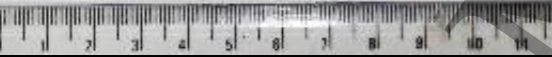

(e) Mesh \#2 50\% Nylon

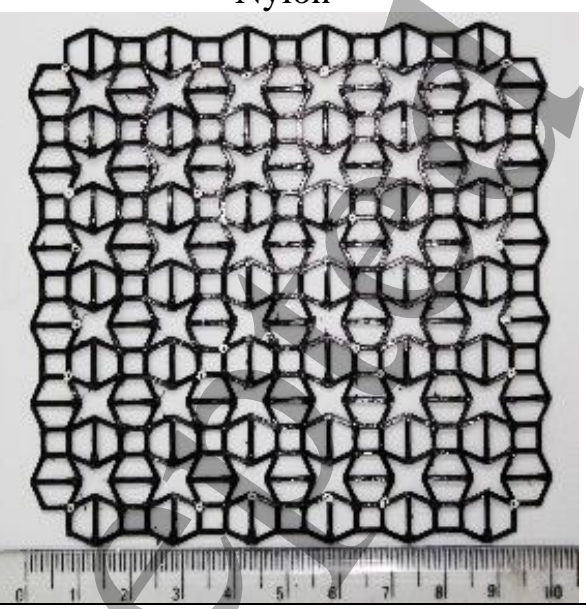

Figure 4 Examples of the meshes tested in the present work. Mesh \#2 50\% in PP is omitted from this representation due to the transparent nature of the material. (b) Mesh \#2

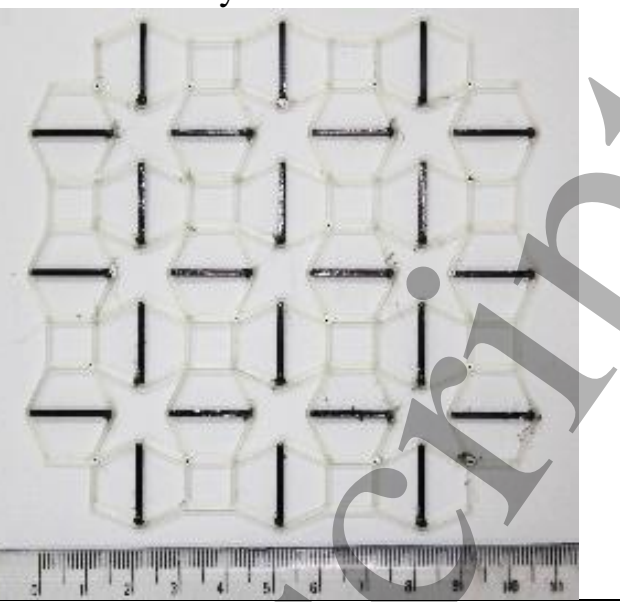

(d) Mesh \#2 50\%

Nylon-PVA
Nylon-PVA

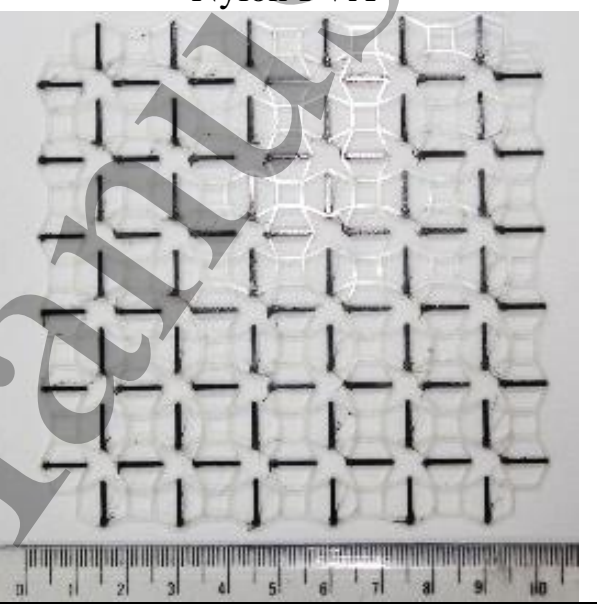

(f) Mesh \#2 50\% $\mathrm{PP}-\mathrm{CPE}+$

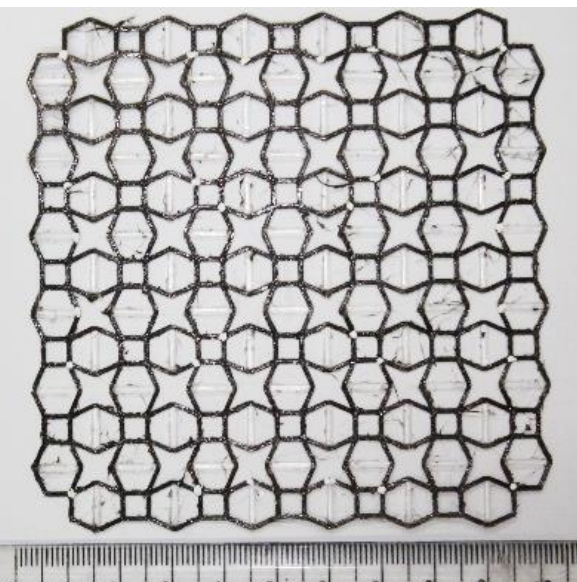

(IIII) 


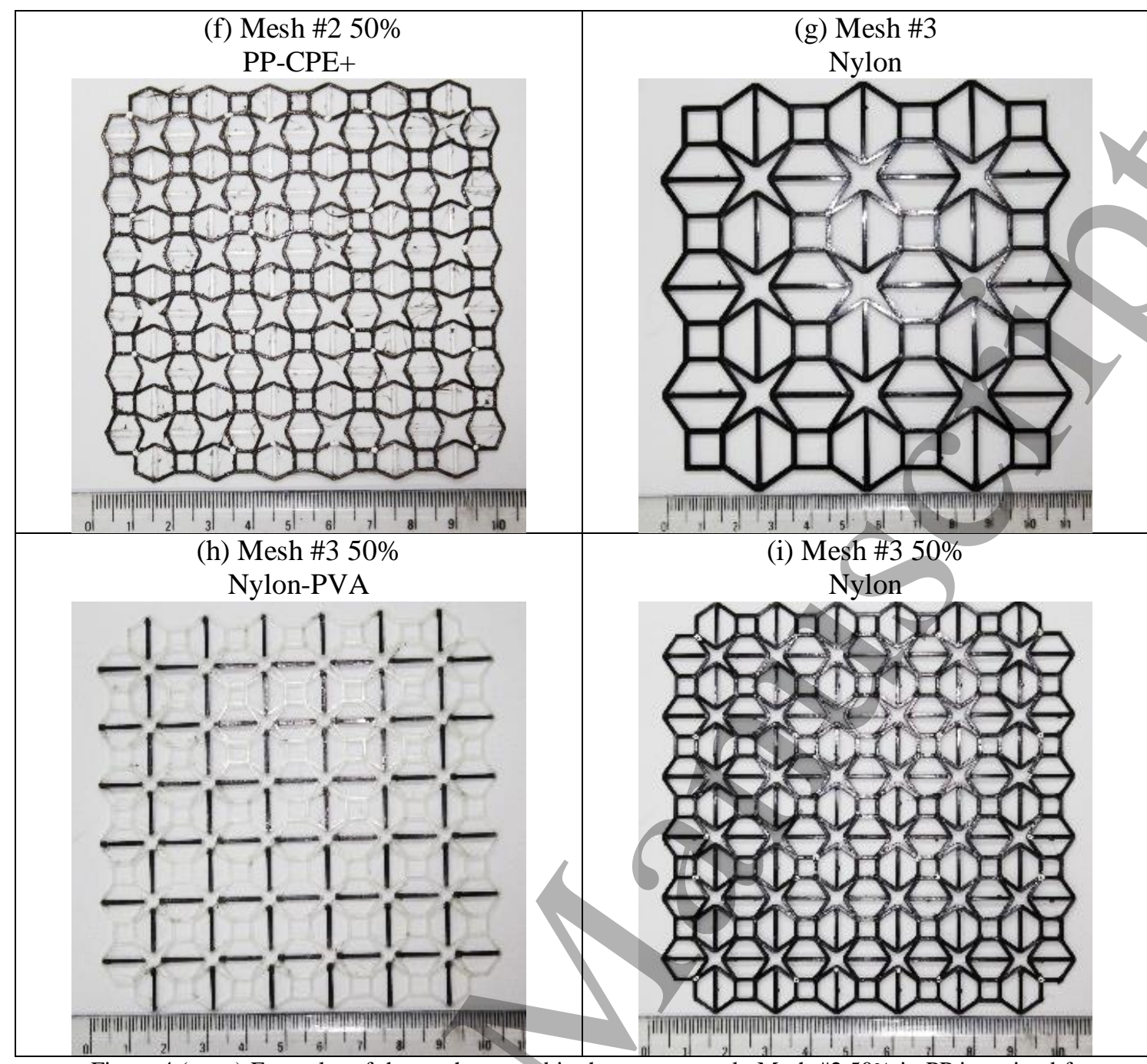

Figure 4 (cont.) Examples of the meshes tested in the present work. Mesh \#2 50\% in PP is omitted from this representation due to the transparent nature of the material.

\section{Results and discussion}

The comparison between the values exhibited by the two \#2 meshes seem attributable to the introduction, in the dual-material case, of a larger proportion of a more rigid material (PVA), which limits the extent of free deformation occurring along the transverse direction while the mesh is being forcefully deformed along the longitudinal direction.

Figure 4 shows the meshes subject to CTE testing. The set of meshes analysed include \#1, \#2, \#2 50\%, \#3 and \#3 50\%, in two dual-material combinations (Nylon-PVA and PP-CPE+) and in single-material versions (Nylon and PP), in accordance with Table 3. Figure 5 presents the measured CTE value for all the meshes considered, in the range 298 to $358 \mathrm{~K}$. The results in figure 5 (b), (d), (f) and (h) provide evidence of effective anepectic behaviour. This result was never attained in any previous research, being the first non-virtual example of a polymer-based composite material exhibiting this type behaviour. In addition, the auxetic behaviour was macroscopically observed for all the meshes studied, confirming that, for the structures studied in the present work, it is indeed inherent to the mesh geometry, depending merely on the structure's re-entrant architecture and not on the material or the materials combinations which constitute it. 


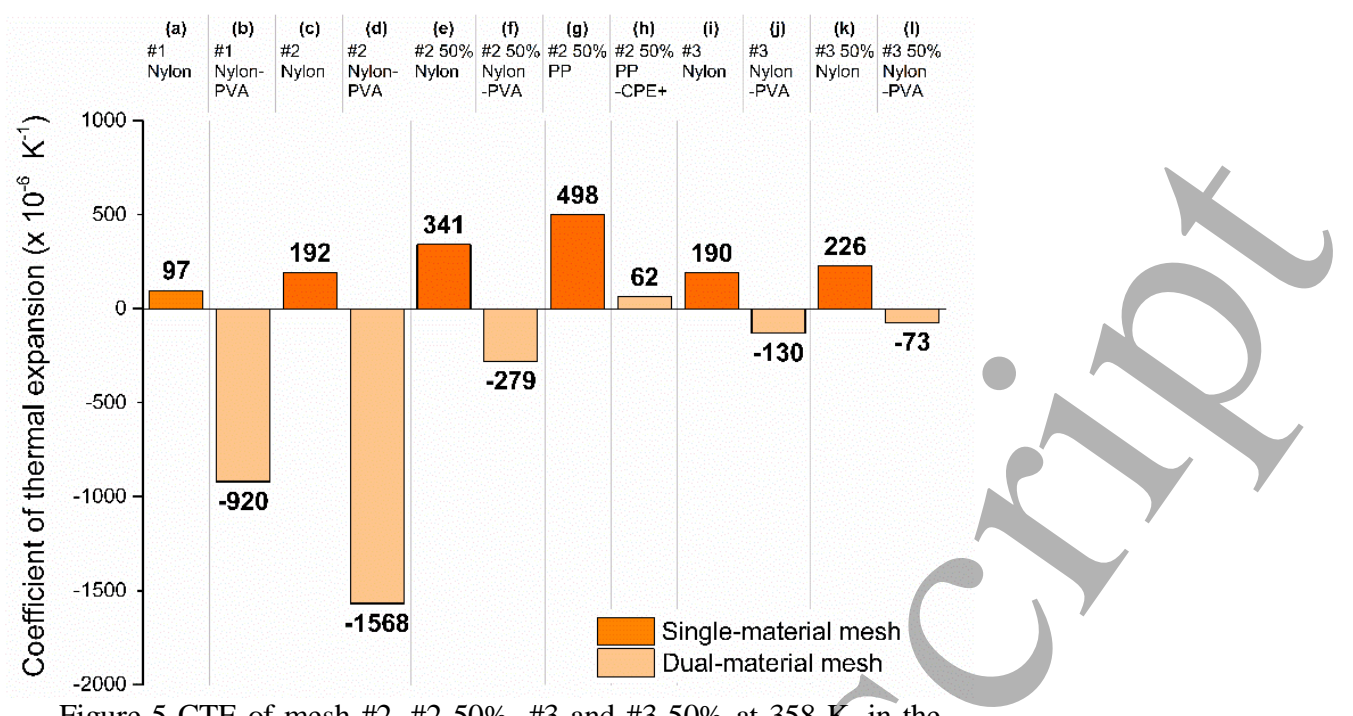

Figure 5 CTE of mesh \#2, \#2 50\%, \#3 and \#3 50\% at $358 \mathrm{~K}$, in the material combinations of Nylon-PVA and PP-CPE+ and in the singlematerial mesh of Nylon and PP. (a) mesh \#1 in Nylon, (b) mesh \#1 in Nylon-PVA, (c) mesh \#2 in Nylon, (d) mesh \#2 in Nylon-PVA, (e) mesh \#2 50\% in Nylon, (f) mesh \#2 50\% in Nylon-PVA, (g) mesh \#2 $50 \%$ in PP, (h) mesh \#2 50\% in PP-CPE+, (i) mesh \#3 in Nylon, (j) mesh \#3 in Nylon-PVA, (k) mesh \#3 50\% in Nylon and (1) mesh \#3 $50 \%$ in Nylon-PVA.

The exact values of PR, shown in Table 4, have been measured only for the case with the more promising CTE value, and for its single-material counterpart.

Table 4 Poisson's ratios determined ${ }^{(*)}$ from elongation tests.

\begin{tabular}{l|c} 
Mesh identification & Poisson's ratio \\
Mesh \#2 Nylon & $v$ \\
Mesh \#2 Nylon-PVA & -0.37 \\
\hline
\end{tabular}

(*) During testing, the meshes were imparted an overall elongation of $10 \mathrm{~mm}$ at room temperature, with a constant elongation rate of 1 $\mathrm{mm} / \mathrm{s}$.

\subsection{Effect of material combination on CTE}

To elucidate how the different material combinations reacted to a temperature change, the meshes displayed in figure 4 were tested. As previously mentioned, the different material combinations selected for this studied were chosen with reference to the criteria predicted by L. Ai et al.[53], as most conducive to an effective NTE behaviour, combining a stiffer and less thermally expansive material with another of more flexible and more thermally expansive behaviour.

In order to ascertain whether the anepectic behaviour was indeed due to the nature of the dualmaterial combinations or if it simply resulted from geometric effects of the structures, there was a need to test the single-material version of the studied meshes, figure 5 (a), (c), (e), (g), (i) and $(\mathrm{k})$, and compare its behaviour with the corresponding dual-material. According to the data in figure 5 (c), when testing mesh \#2 with a single-material CTE is positive $\left(192 \times 10^{-6} \mathrm{~K}^{-1}\right)$, whereas when testing a dual-material mesh (in Nylon-PVA) the CTE becomes negative $\left(-1568 \times 10^{-6}\right.$ $\mathrm{K}^{-1}$ ), which confirms the previously reported observations. Similar results occurred with mesh \#2 50\%: for Nylon the measured CTE (figure 5 (e)) is positive $\left(341 \times 10^{-6} \mathrm{~K}^{-1}\right)$ but becomes negative for the equivalent dual-material (Nylon-PVA) mesh $\left(-279 \times 10^{-6} \mathrm{~K}^{-1}\right)$, showing that the anepectic 
behaviour is confined to the composite meshes, similar effect is noted for mesh \#1. The results in figure 5 (e), (f), (g) and (h) illustrate a similar effect, for mesh \#3 and mesh \#3 50\%. When testing the single-material mesh \#3 (in Nylon) the CTE is positive for both the unaltered \#3 mesh $(190 \times$ $\left.10^{-6} \mathrm{~K}^{-1}\right)$ and the halved, \#3 50\% mesh in Nylon $\left(226 \times 10^{-6} \mathrm{~K}^{-1}\right)$ and negative for the equivalent dual-material (Nylon-PVA) mesh $\left(-130 \times 10^{-6} \mathrm{~K}^{-1}\right.$ and $-73 \times 10^{-6} \mathrm{~K}^{-1}$, respectively). Consequently, when studying the single-material versions of the aforementioned meshes the CTE values were all positive, and the equivalent dual-material meshes all reached negative CTE values, providing evidence of the success in fabricating anepectic dual-material meshes, from constituent materials with positive coefficients of thermal expansion.

By way of contrast, figure 5 (g) shows, that testing mesh \#2 50\% with a single-material (in $\mathrm{PP}$ ) the CTE was positive, $498 \times 10^{-6} \mathrm{~K}^{-1}$ but when testing the dual-material mesh (in PP-CPE+) the CTE remained positive, albeit with a smaller value of $62 \times 10^{-6} \mathrm{~K}^{-1}$ (figure (h)), showing that the combination of PP-CPE+ did not succeed in having an anepectic behaviour. This shows that the anepectic behaviour is only achieved through the adequate material combination in the designed mesh. The relation between the CTE and the Young's moduli of the constituent materials in the composite mesh must be precise in order to result in the effective deformation of the more flexible constituent by the stiffer one when both dilate during heating, resulting in the overall mesh contraction by way of its particular geometry. For the case under analysis, while the stiffness ratios are similar $\left(\mathrm{E}_{\mathrm{Nylon}} / \mathrm{E}_{\mathrm{PVA}}=0.38\right.$ vs. $\left.\mathrm{E}_{\mathrm{PP}} / \mathrm{E}_{\mathrm{CPE}+}=0.15\right)$ the ratio of the $\mathrm{CTE}$ values is much larger (8.1) for the Nylon-PVA combination than for PP-CPE+ (for which it presents a value of 3.5). Thus, the requirement to anepectic behaviour seems to be that the combined materials exhibit similar (but different) stiffness but widely differing thermal expansion. Provided this requirement is respected, a choice of materials conducive to slight changes of the exact values of the stiffness and CTE ratios should allow fine tuning of the resulting anepectic behaviour.

\subsection{Effect of mesh architecture on CTE}

After ascertaining the importance of the adequate material combination to the emergence of the anepectic behaviour, the effectiveness of each geometry needed to be elucidated. This comparison is performed for meshes \#1, \#2 and \#3 in Nylon-PVA, the resulting CTE values being presented in figure 5 (b), (d) and (j). A portion of meshes tested are displayed in figure 4 (a) and (b).

As can be observed, all chosen dual-material meshes attained a negative coefficient of thermal expansion, mesh \#2 being the extreme case, showing an extreme value of $-1568 \times 10^{-6} \mathrm{~K}^{-1}$ when the temperature varied between room temperature and $358 \mathrm{~K}$, (figure 5 (d)). Overall, the results in figure 5 provide evidence of tuneable anepectic behaviour.

In particular, the CTE value first decreases significantly with the increase of the angle $\theta$, which changes from $15^{\circ}$ in mesh \#1 to $25^{\circ}$ in mesh \#2, (showed in Table 1), reaching an extreme value, after which the CTE again increases as $\theta$ progresses to $35^{\circ}$ in mesh \#3. This implies that an angle of $25^{\circ}$ (or around this value) is more beneficial in attaining a more negative CTE, in agreement with predictions by L. Ai et al. [53]. Once more, the observed behaviour reveals the possibility of tuning the magnitude of the anepectic behaviour.

\subsection{Effect of mesh scale on CTE}

In order to identify how the anepectic effect is influenced by the mesh size, meshes \#2 and \#3, either single-material (Nylon) and dual-material (Nylon-PVA), were compared, for both the fulland half-scale sizes.

Sensitivity differences between the two designs are already evident from the single-material meshes: while the CTE for \#2 Nylon mesh changes from a full-size (figure 5 (c)) $192 \times 10^{-6} \mathrm{~K}^{-1}$ 
to a half-size (figure 5 (e)) $341 \times 10^{-6} \mathrm{~K}^{-1}$, which corresponds to a $78 \%$ change, for mesh \#3 the values are respectively $190 \times 10^{-6} \mathrm{~K}^{-1}$ (full-size, figure 5 (i)) and $226 \times 10^{-6} \mathrm{~K}^{-1}$ (half-size, figure $5(\mathrm{k}))$, resulting in a $19 \%$ variation.

Such differences are exacerbated for the dual-meshes, where a size reduction of $50 \%$ led to negative CTE values for all dual-material meshes, although no generalized improvement could be observed: compared to the full size meshes, the size reduction led to a less markedly negative CTE. Again, this offers a different choice to fine-tune the anepectic behaviour. Peering over the CTE values measured for the different cases of Nylon-PVA meshes, one is also led to conclude that mesh \#2, which at full-size scale (figure 5 (d)) exhibits the highest magnitude negative CTE, at $-1568 \times 10^{-6} \mathrm{~K}^{-1}$, is the most sensitive to scale of the two geometries under comparison, CTE changing to $-279 \times 10^{-6} \mathrm{~K}^{-1}$ when the mesh is halved (figure 5 (f)), corresponding to a $82 \%$ change. By comparison, the relative change in CTE for mesh \#3 is smaller, from a full-size (figure 5 (j)) $-130 \times 10^{-6} \mathrm{~K}^{-1}$ to a half-size (figure 5 (l)) $-73 \times 10^{-6} \mathrm{~K}^{-1}$, a $44 \%$ change.

The above observations show that a larger value of length parameter (for values around 10 $\mathrm{mm}$ for $\mathrm{H}_{1}$ and $\mathrm{H}_{2}$ and $1 \mathrm{~mm}$ for $\mathrm{t}$, corresponding to the full size meshes) is more beneficial for attaining a more negative CTE, providing evidence of the effect of scaling on a unit cell in achieving and tuning anepectic behaviour through mesh design.

\subsection{Effect of plastic flow during heating}

Mechanical properties of polymeric materials are highly sensitive to temperature; particularly above glass transition temperature $\left(\mathrm{T}_{\mathrm{g}}\right)$ all polymers will show plastic deformation. Therefore, the influence of this plastic deformation on the response of the anepectic meshes must be clarified. For that purpose, two different material combinations were tested, using half-size mesh \#2. While Nylon-PVA was chosen due to having close $T_{\mathrm{g}}$ values, PP-CPE+ was selected because the two materials exhibit widely differing $\mathrm{T}_{\mathrm{g}}$ values, as shown in Table 2 above. For the sake of comparison, the single-material case, based on PP, was also tested. The CTE values for these meshes are presented in figure 6 . The observation of these results firstly confirms that, irrespective of being below or above $\mathrm{T}_{\mathrm{g}}$, single-material meshes never exhibit anepectic behaviour. On the other hand, for the dual-material cases, anepectic behaviour only manifests itself above $T_{g}$ of both constituents.

After completing the tests above, the meshes were examined and found to have undergone plastic deformation, due to the polymer flow occurring above $\mathrm{T}_{\mathrm{g}}$. This prompted the execution of sequential heating and cooling tests, which were performed on mesh \#2 50\% in Nylon-PVA, to elucidate the effect on the anepectic behaviour of the plastic deformation of the elementary struts; the relevant results are shown in figure 7. 


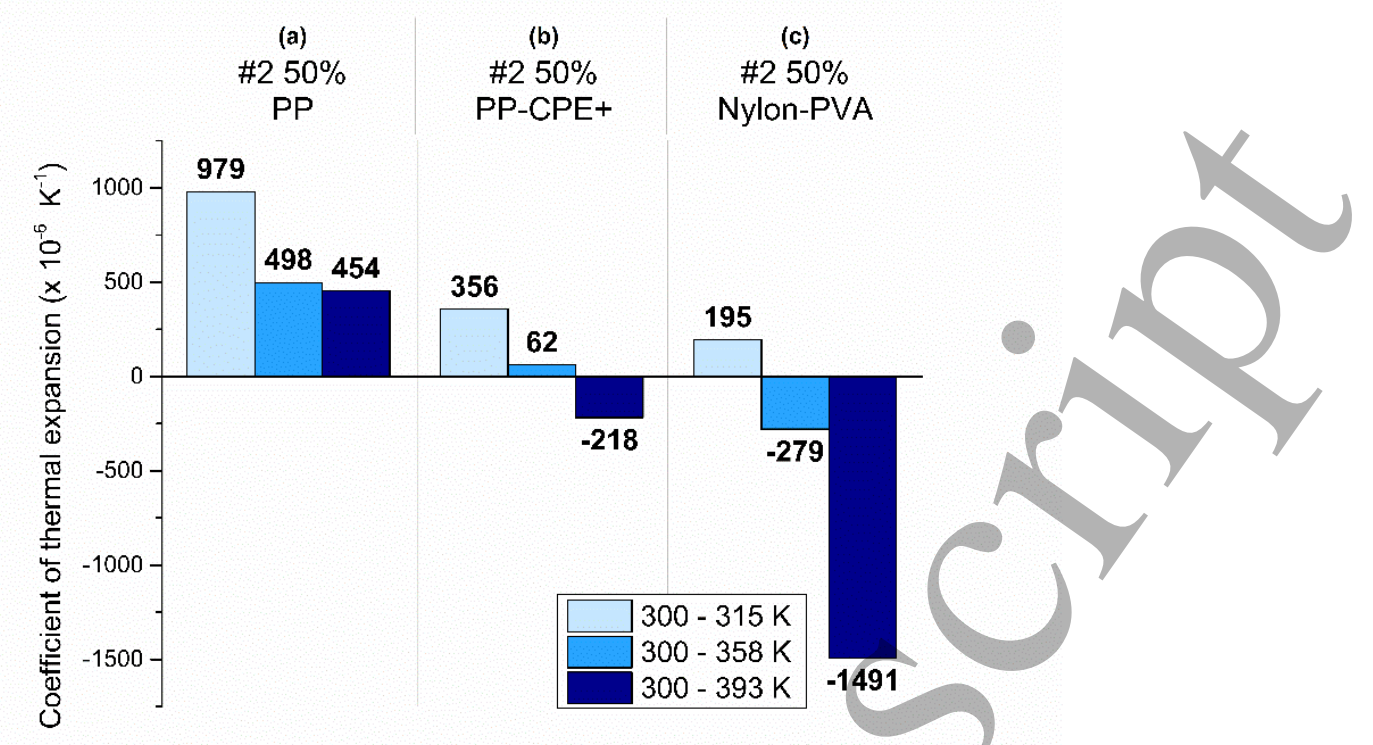

Figure $6(\mathrm{a}, \mathrm{b}) \mathrm{CTE}$ of mesh \#2 50\% from the variation of $300^{\circ} \mathrm{C}$ until $393{ }^{\circ} \mathrm{C}$, in the material combination of PP-CPE+ and in the single-material mesh of PP. (c) CTE of mesh \#2 50\% from the variation of $300{ }^{\circ} \mathrm{C}$ until $393 \mathrm{~K}$, in the material combination of Nylon-PVA.

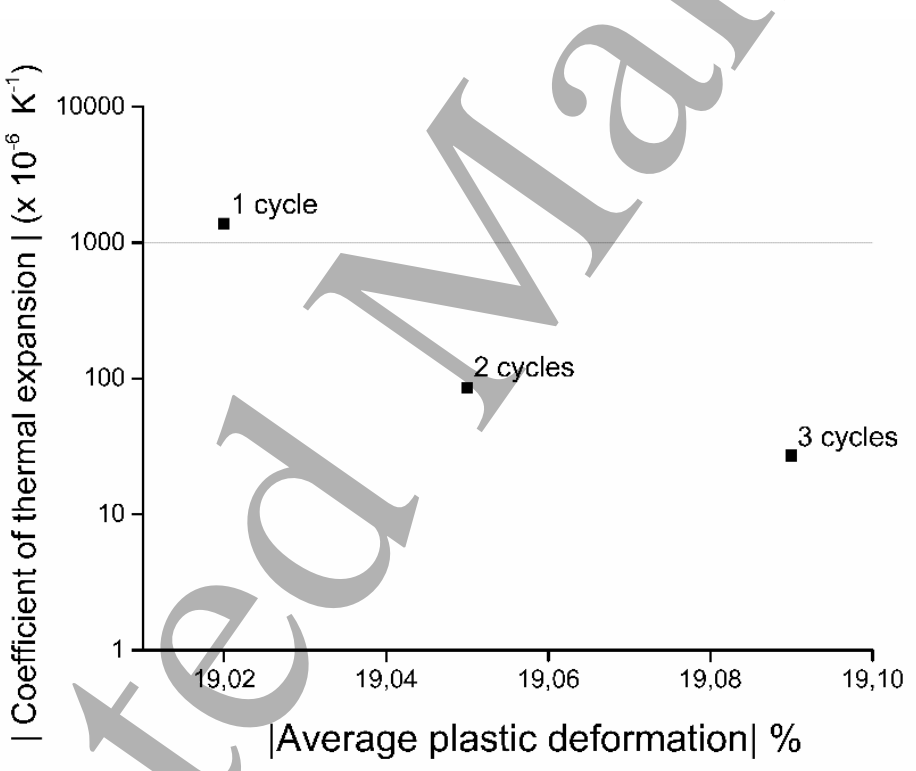

Figure 7 Variation of the measured value of the coefficient of thermal expansion for the Nylon-PVA \#2 50\% mesh, as the plastic deformation increases during consecutive heating and cooling cycles; the initial dimensions of the mesh (before heating) were taken as reference for the calculation of the average plastic deformation.

The trend exhibited by the results shows that the anepectic effect becomes progressively less pronounced, probably due to the accumulation of irreversible deformation. This effect is most noticeable already after the first heating and cooling sequence, so that when the mesh undergoes subsequent heating the anepectic behaviour has become negligible.

Ultimately the anepectic effect and its possible tuneability stems from an adequate design of the mesh, accurate selection and combination of material and the synergy of the plastic flow of the constituents occurring above $\mathrm{T}_{\mathrm{g}}$, which will bring about the desired designed deformation. 


\section{Conclusion}

A series of polymeric metamaterials with tuneable mechanical properties were designed, using star-shaped re-entrant bi-dimensional structures, utilizing constituents with positive thermal expansion, and were subsequently fabricated with a 3-D printing system, being tested so as to simultaneously display negative values of Poisson ratio and CTE, configuring an anepectic behaviour. Thus, to the authors' knowledge, the present work provides the first non-virtual example of a polymer-based composite material exhibiting anepectic behaviour.

Design variables for each mesh consisted of constituent materials properties and combinations, three different length parameters and one angular parameter. The effects of these geometrical parameters and material combinations on the CTE and Poisson's ratio were studied through a series of room temperature active deformation and controlled heating passive deformation tests.

The anepectic behaviour could only be observed when the combined materials presented similar, yet different, stiffness values, together with widely different intrinsic CTE, the stiffer and less thermally expansive material forming a continuous re-entrant network, with connecting struts between contiguous stars made from the second material. Provided this requirement is respected, a choice of materials conducive to slight changes of the exact values of the stiffness and CTE ratios should allow fine tuning of the resulting anepectic behaviour. Furthermore, the anepectic behaviour was only observed above the glass transition temperature of both constituent materials. Due to the irreversible nature of the deformation occurring at such temperature ranges, when subjected to consecutive heating and cooling cycles, the anepectic effect displayed by the meshes became progressively less pronounced.

Geometry-wise, the observed CTE showed a marked dependence on the angular parameter, attaining a more negative value for an intermediate angle around $25^{\circ}$. Concerning the length parameters, the CTE became more negative for larger sized meshes.

Ultimately the anepectic effect and the possibility for fine tuning stems from an adequate design of the mesh, accurate selection and combination of materials and the synergy of the plastic flow of the constituents occurring above $\mathrm{T}_{\mathrm{g}}$, which will bring about the desired designed deformation.

Design and optimization of anepectic structures, with appropriately selected geometrical parameters and material combination, deserve further investigation. The models, materials and printing technology are expected to evolve further, and a great deal of work and research needs to be done by the scientific community on this matter. The goal is to utilise these meshes in to the biomaterial field and they shall be tuned to work at the working temperature range of the human body. This paper should open a path into further studies concerning polymer-based anepectic structures, mainly for the biomedical field, but the creation of metallic anepectic structures, e.g. for the aerospace and defence industry, also becomes a foreseeable prospect.

\section{Acknowledgements}

This work was partially funded by FEDER funds through the COMPETE 2020 Program and National Funds through FCT - Portuguese Foundation for Science and Technology under the project UID/CTM/50025/2013.

\section{References}


[1] J. N. Grima, P.-S. Farrugia, R. Gatt, and V. Zammit, "Connected Triangles Exhibiting Negative Poisson's Ratios and Negative Thermal Expansion," J. Phys. Soc. Japan, vol. 76, no. 2, p. 025001, Feb. 2007.

[2] C. S. Ha, E. Hestekin, J. Li, M. E. Plesha, and R. Lakes, "Controllable thermal expansion of large magnitude in chiral negative Poisson's ratio lattices," Phys. status solidi, vol. 252, no. 7, pp. 1431-1434, Jul. 2015.

[3] X. Ren, J. Shen, A. Ghaedizadeh, H. Tian, and Y. Min Xie, "Experiments and parametric studies on 3D metallic auxetic metamaterials with tuneable mechanical properties," Smart Mater. Struct., vol. 24, no. 9, p. 095016, Sep. 2015.

[4] K. Takenaka, "Negative thermal expansion materials: technological key for control of thermal expansion," Sci. Technol. Adv. Mater., vol. 13, no. 1, p. 013001, Feb. 2012.

[5] G. D. Barrera, J. A. O. Bruno, T. H. K. Barron, and N. L. Allan, "Negative thermal expansion," J. Phys. Condens. Matter, vol. 17, no. 4, pp. R217-R252, Feb. 2005.

[6] W. Miller, C. W. Smith, D. S. Mackenzie, and K. E. Evans, "Negative thermal expansion: a review," J. Mater. Sci., vol. 44, no. 20, pp. 5441-5451, Oct. 2009.

[7] C. Closmann, A. W. Sleight, and J. C. Haygarth, "Low-Temperature Synthesis of ZrW2O8and Mo-Substituted ZrW2O8," J. Solid State Chem., vol. 139, no. 2, pp. 424426, Sep. 1998.

[8] W. J. Clegg and A. Kelly, "Choosing Materials for Composites with a Greater Range of Properties," Adv. Eng. Mater., vol. 4, no. 6, pp. 388-392, Jun. 2002.

[9] N. Yamamoto, E. Gdoutos, R. Toda, V. White, H. Manohara, and C. Daraio, "Thin Films with Ultra-low Thermal Expansion," Adv. Mater., vol. 26, no. 19, pp. 3076-3080, May 2014.

[10] A. W. Sleight, "Negative thermal expansion materials," Curr. Opin. Solid State Mater. Sci., vol. 3, no. 2, pp. 128-131, Apr. 1998.

[11] A. Versluis, W. H. Douglas, and R. L. Sakaguchi, "Thermal expansion coefficient of dental composites measured with strain gauges," Dent. Mater., vol. 12, no. 5, pp. 290294, 1996.

[12] L. Wu, B. Li, and J. Zhou, "Isotropic Negative Thermal Expansion Metamaterials," ACS Appl. Mater. Interfaces, vol. 8, no. 27, pp. 17721-17727, Jul. 2016.

[13] K. E. Evans and A. Alderson, "Auxetic Materials: Functional Materials and Structures from Lateral Thinking!," Adv. Mater., vol. 12, no. 9, pp. 617-628, May 2000.

[14] E. O. Dickerson and B. DiMartino, "Off-Axis Strength and Testing of Filamentary Materials for Aircraft Application," Adv. Fibrous Reinf. Compos., vol. 10, p. H-23, 1966.

[15] B. M. LEMPRIERE, "Poisson's ratio in orthotropic materials.," AIAA J., vol. 6, no. 11, pp. 2226-2227, Nov. 1968.

[16] L. J. Gibson, “The Elastic And Plastic Behaviour Of Cellular Materials,” University of cambridge, 1981.

[17] L. J. Gibson, M. F. Ashby, G. S. Schajer, and C. I. Robertson, "The Mechanics of TwoDimensional Cellular Materials," Proc. R. Soc. A Math. Phys. Eng. Sci., vol. 382, no. 1782, pp. 25-42, Jul. 1982. 
[18] R. Lakes, "Foam Structures with a Negative Poisson's Ratio," Science (80-. )., vol. 235, no. 4792, pp. 1038-1040, Feb. 1987.

[19] K. E. Evans, M. A. Nkansah, I. J. Hutchinson, and S. C. Rogers, "Molecular network design," Nature, vol. 353, p. 124, Sep. 1991.

[20] M. H. Fu, O. T. Xu, L. L. Hu, and T. X. Yu, "Nonlinear shear modulus of re-entrant hexagonal honeycombs under large deformation," Int. J. Solids Struct., vol. 80, pp. 284296, Feb. 2016.

[21] C. Huang and L. Chen, "Negative Poisson's Ratio in Modern Functional Materials," Adv. Mater., vol. 28, no. 37, pp. 8079-8096, Oct. 2016.

[22] R. Lakes, Roderic SLakes, "Negative-Poisson's-Ratio Materials: Auxetic Solids," Annu. Rev. Mater. Res., vol. 47, no. 1, pp. 63-81, Jul. 2017.

[23] M. Shokri Rad, Y. Prawoto, and Z. Ahmad, "Analytical solution and finite element approach to the 3D re-entrant structures of auxetic materials," Mech. Mater., vol. 74, pp. 76-87, Jul. 2014.

[24] K. K. Saxena, R. Das, and E. P. Calius, "Three Decades of Aux etics' Research Materials with Negative Poisson's Ratio: A Review," Adv. Eng. Mater., vol. 18, no. 11, pp. 1847-1870, Nov. 2016.

[25] X.-T. Wang, B. Wang, X.-W. Li, and L. Ma, "Mechanical properties of 3D re-entrant auxetic cellular structures," Int. J. Mech. Sci., vol. 131-132, no. March, pp. 396-407, Oct. 2017.

[26] M. Schenk and S. D. Guest, "Geometry of Miura-folded metamaterials," Proc. Natl. Acad. Sci., vol. 110, no. 9, pp. 3276-3281, Feb. 2013.

[27] S. Babaee, J. Shim, J. C. Weaver, E. R. Chen, N. Patel, and K. Bertoldi, "3D Soft Metamaterials with Negative Poisson's Ratio," Adv. Mater., vol. 25, no. 36, pp. 50445049, Sep. 2013.

[28] O. Bouaziz, J. P. Masse, S. Allain, L. Orgéas, and P. Latil, "Compression of crumpled aluminum thin foils and comparison with other cellular materials," Mater. Sci. Eng. A, vol. 570, pp. 1-7, May 2013.

[29] T. Strek, H. Jopek, E. Idczak, and K. Wojciechowski, "Computational Modelling of Structures with Non-Intuitive Behaviour," Materials (Basel)., vol. 10, no. 12, p. 1386, Dec. 2017.

[30] C. S. Ha, M. E. Plesha, and R. Lakes, "Chiral three-dimensional isotropic lattices with negative Poisson's ratio,” Phys. status solidi, vol. 253, no. 7, pp. 1243-1251, Jul. 2016.

[31] D. Rodney, B. Gadot, O. R. Martinez, S. R. du Roscoat, and L. Orgéas, "Reversible dilatancy in entangled single-wire materials," Nat. Mater., vol. 15, no. 1, pp. 72-77, Jan. 2016.

[32] G. Carta, M. Brun, and A. Baldi, "Design of a porous material with isotropic negative Poisson's ratio," Mech. Mater., vol. 97, pp. 67-75, Jun. 2016.

[33] A. A. Poźniak, K. W. Wojciechowski, J. N. Grima, and L. Mizzi, "Planar auxeticity from elliptic inclusions," Compos. Part B Eng., vol. 94, no. 2, pp. 379-388, Jun. 2016.

[34] H. Jopek and T. Strek, "Thermal and structural dependence of auxetic properties of composite materials," Phys. status solidi, vol. 252, no. 7, pp. 1551-1558, Jul. 2015. 
[35] H. Jopek and T. Stręk, "Thermoauxetic Behavior of Composite Structures," Materials (Basel)., vol. 11, no. 2, p. 294, Feb. 2018.

[36] K. Wang, Y.-H. Chang, Y. Chen, C. Zhang, and B. Wang, "Designable dual-material auxetic metamaterials using three-dimensional printing," Mater. Des., vol. 67, pp. 159 164, Feb. 2015.

[37] Q. Wang, J. A. Jackson, Q. Ge, J. B. Hopkins, C. M. Spadaccini, and N. X. Fang, "Lightweight Mechanical Metamaterials with Tunable Negative Thermal Expansion," Phys. Rev. Lett., vol. 117, no. 17, p. 175901, Oct. 2016.

[38] R. Gatt and J. N. Grima, "Negative compressibility," Phys. status solidi - Rapid Res. Lett., vol. 2, no. 5, pp. 236-238, Oct. 2008.

[39] K. K. Dudek, D. Attard, R. Caruana-Gauci, K. W. Wojciechowski, and J. N. Grima, "Unimode metamaterials exhibiting negative linear compressibility and negative thermal expansion,” Smart Mater. Struct., vol. 25, no. 2, p. 025009, Feb. 2016.

[40] J. N. Grima, P. S. Farrugia, R. Gatt, and V. Zammit, "A system with adjustable positive or negative thermal expansion," Proc. R. Soc. A Math. Phys. Eng. Sci., vol. 463, no. 2082, pp. 1585-1596, 2007.

[41] J. B. Hopkins, K. J. Lange, and C. M. Spadaccini, "Designing Microstructural Architectures With Thermally Actuated Properties Using Freedom, Actuation, and Constraint Topologies," J. Mech. Des., vol. 135, no. 6, p. 061004, May 2013.

[42] J. B. Hopkins, Y. Song, H. Lee, N. X. Fang, and C. M. Spadaccini, "Polytope SectorBased Synthesis and Analysis of Microstructural Architectures With Tunable Thermal Conductivity and Expansion," J. Mech. Des., vol. 138, no. 5, p. 051401, Mar. 2016.

[43] R. Lakes, "Cellular solid structures with unbounded thermal expansion," J. Mater. Sci. Lett., vol. 15, no. 6, pp. 475-477, 1996.

[44] R. Lakes, "Cellular solids with tunable positive or negative thermal expansion of unbounded magnitude," Appl. Phys. Lett., vol. 90, no. 22, p. 221905 , May 2007.

[45] W. Miller, D. S. Mackenzie, C. W. Smith, and K. E. Evans, "A generalised scaleindependent mechanism for tailoring of thermal expansivity: Positive and negative," Mech. Mater., vol. 40, no. 4-5, pp. 351-361, 2008.

[46] K. Wei, H. Chen, Y. Pei, and D. Fang, "Planar lattices with tailorable coefficient of thermal expansion and high stiffness based on dual-material triangle unit," J. Mech. Phys. Solids, vol. 86, pp. 173-191, Jan. 2016.

[47] H. Xu and D. Pasini, "Structurally Efficient Three-dimensional Metamaterials with Controllable Thermal Expansion," Sci. Rep., vol. 6, no. 1, p. 34924, Dec. 2016.

[48] J. Qu, M. Kadic, A. Naber, and M. Wegener, "Micro-Structured Two-Component 3D Metamaterials with Negative Thermal-Expansion Coefficient from Positive Constituents," Sci. Rep., vol. 7, no. 1, p. 40643, Dec. 2017.

[49] A. Takezawa, M. Kobashi, and M. Kitamura, "Porous composite with negative thermal expansion obtained by photopolymer additive manufacturing," APL Mater., vol. 3, no. 7, p. 076103, Jul. 2015.

[50] C. A. Steeves, S. L. dos Santos e Lucato, M. He, E. Antinucci, J. W. Hutchinson, and A. G. Evans, "Concepts for structurally robust materials that combine low thermal expansion with high stiffness," J. Mech. Phys. Solids, vol. 55, no. 9, pp. 1803-1822, Sep. 
2007.

[51] J. Berger, C. Mercer, R. M. McMeeking, and A. G. Evans, "The Design of Bonded Bimaterial Lattices that Combine Low Thermal Expansion with High Stiffness," J. Am. Ceram. Soc., vol. 94, pp. s42-s54, Jun. 2011.

[52] Y. Ding and R. Kovacevic, "Feasibility Study on 3-D Printing of Metallic Structural Materials with Robotized Laser-Based Metal Additive Manufacturing," Jom, vol. 68, no. 7, pp. 1774-1779, 2016.

[53] L. Ai and X.-L. Gao, "Metamaterials with negative Poisson's ratio and non-positive thermal expansion," Compos. Struct., vol. 162, pp. 70-84, Feb. 2017.

[54] O. Sigmund and S. Torquato, "Composites with extremal thermal expansion coefficients,” Appl. Phys. Lett., vol. 69, no. 21, pp. 3203-3205, 1996.

[55] P. S. Theocaris, G. E. Stavroulakis, and P. D. Panagiotopoulos, "Negative Poisson's ratios in composites with star-shaped inclusions: a numerical homogenization approach," Arch. Appl. Mech. (Ingenieur Arch., vol. 67, no. 4, pp. 274-286, Apr. 1997.

[56] X. Gong, J. Huang, F. Scarpa, Y. Liu, and J. Leng, "Zero Poisson's ratio cellular structure for two-dimensional morphing applications," Compos. Struct., vol. 134, pp. 384-392, Dec. 2015. 